\title{
Diversity and Composition of Soil Microorganisms between Abandoned and Selective-Farming Farmlands in an Antimony Mining Area
}

\author{
Yihuan Du ${ }^{1}$, Renyan Duan ${ }^{1}$, Yaqi Zhang ${ }^{1}$, Li Yang ${ }^{1}$, Yuxiang Lin ${ }^{1}$, and Luo Yucai ${ }^{1}$ \\ ${ }^{1}$ Hunan University of Humanities Science and Technology
}

January 24, 2022

\begin{abstract}
Background and Aims Land abandonment and selective farming are two common management methods to restore the soil conditions of low pollution farmland in mining areas. The soil microbial community plays an important role in farmland soil restoration, however, few studies have compared the composition and diversity of soil microorganisms between the abandoned farmlands (AFS) and selective-farming farmlands (FFS). Here, the effects of AFS and FFS on soil properties and microbial diversity were evaluated in an antimony ( $\mathrm{Sb}$ ) mining area in southern China. This study aimed to identify effective land management methods in terms of positive or negative changes in soil environment and bacterial diversity. Results Due to the influence of long-term fertilization, the nutritional properties (e.g., available N, P, and K) of soil in FFS were significantly improved and the microbial alpha diversity including Chao1 index, Simpson index, Shannon index, and Pielou-e index significantly increased, compared to AFS. At the phylum level, FFS had higher relative abundances of Chloroflexi, Acidobacteria, Gemmatimonadetes, and Rokubacteria, and lower relative abundances of Firmicutes, Actinobacteria, and Bacteroidetes. At the genus level, FFS had higher relative abundances of Acidothermus, Haliangium, and Rokubacteriales, and lower relative abundances of Bacillus, Rhodococcus, Sphingomonas, and 67-14. Redundancy analysis indicated that soil heavy metal content and soil fertility were closely correlated with the soil microbial community. Altogether, selective farming of low pollution farmland in the mining area can improve soil properties and soil microbial diversity.
\end{abstract}

\section{Hosted file}

000_Abandoned_and_Selective_Farming_Farmland_duan-1-17.docx available at https://authorea. com/users/457087/articles/553951-diversity-and-composition-of-soil-microorganisms-

between-abandoned-and-selective-farming-farmlands-in-an-antimony-mining-area

\section{Hosted file}

Table 1.doc available at https://authorea.com/users/457087/articles/553951-diversity-andcomposition-of-soil-microorganisms-between-abandoned-and-selective-farming-farmlands-inan-antimony-mining-area 

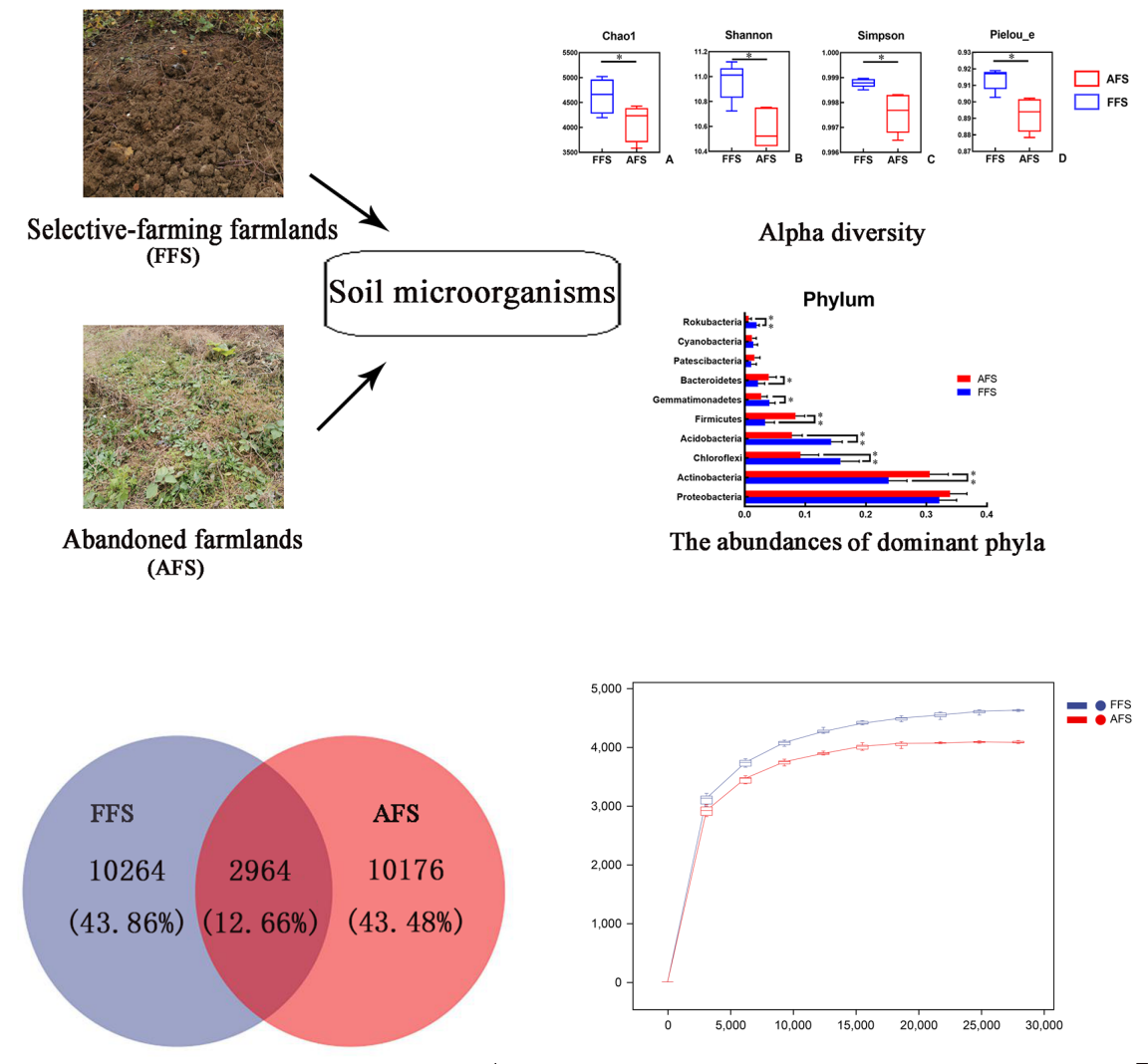

A
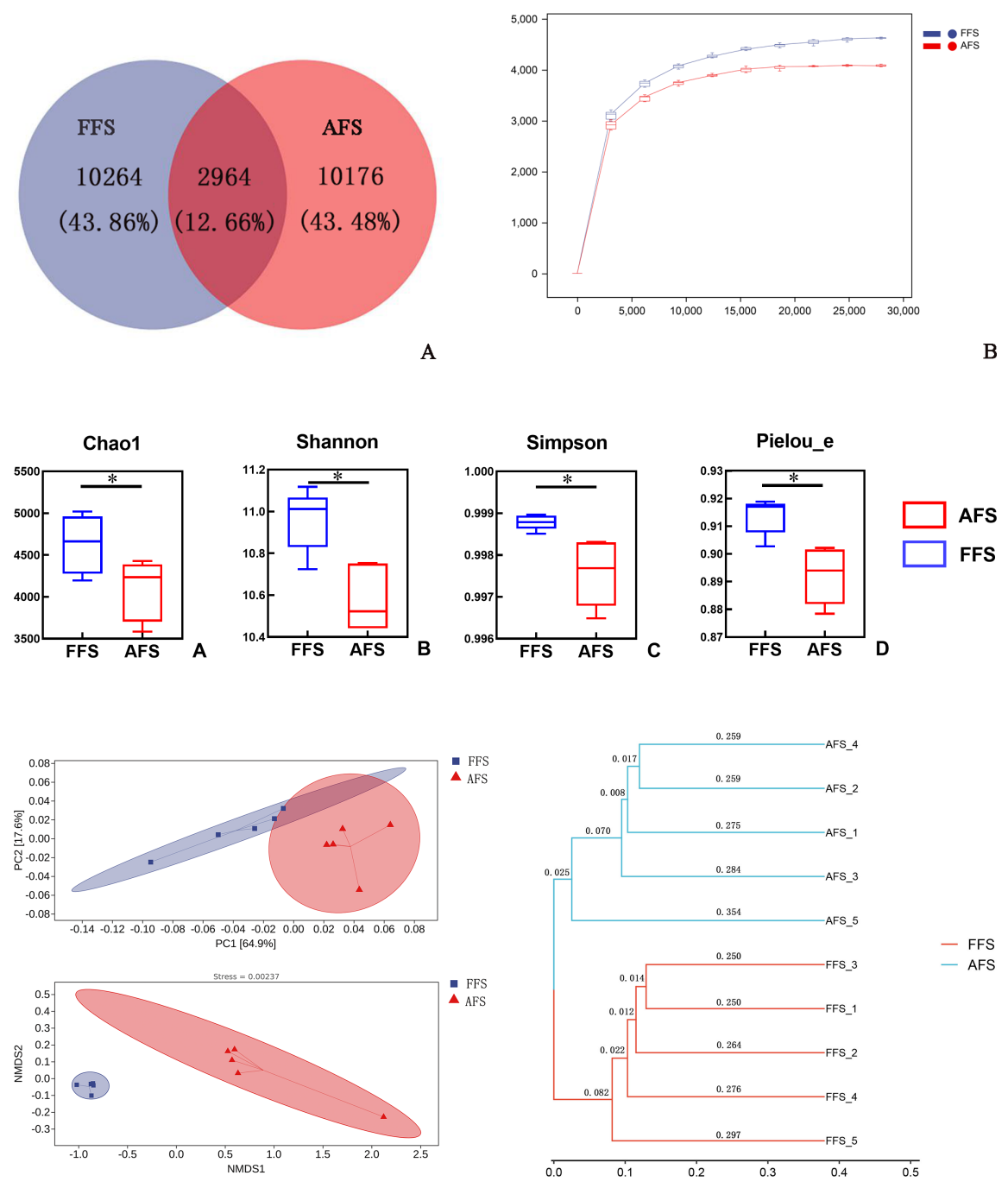
Phylum
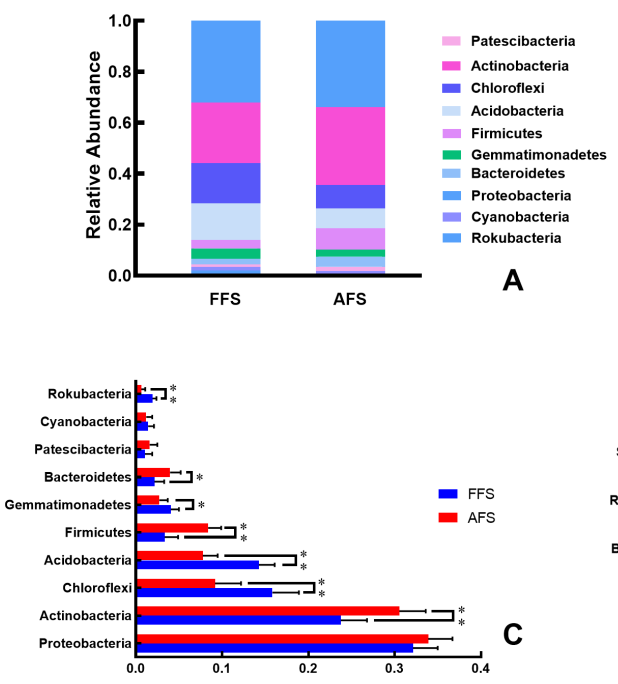

Genus
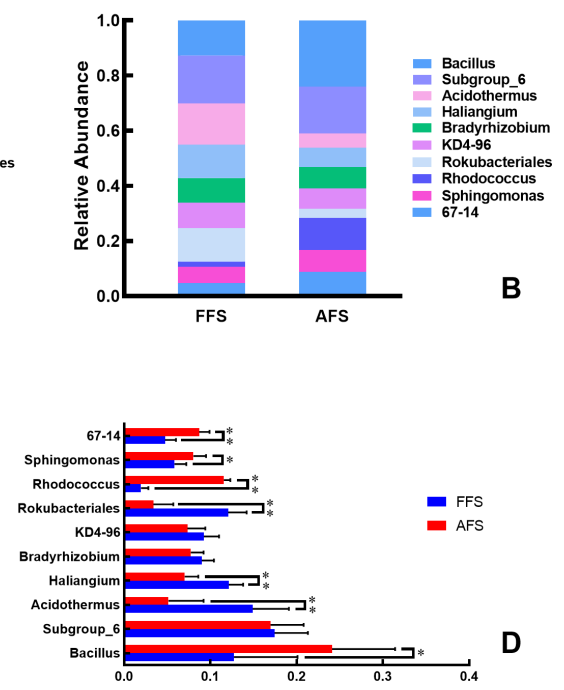


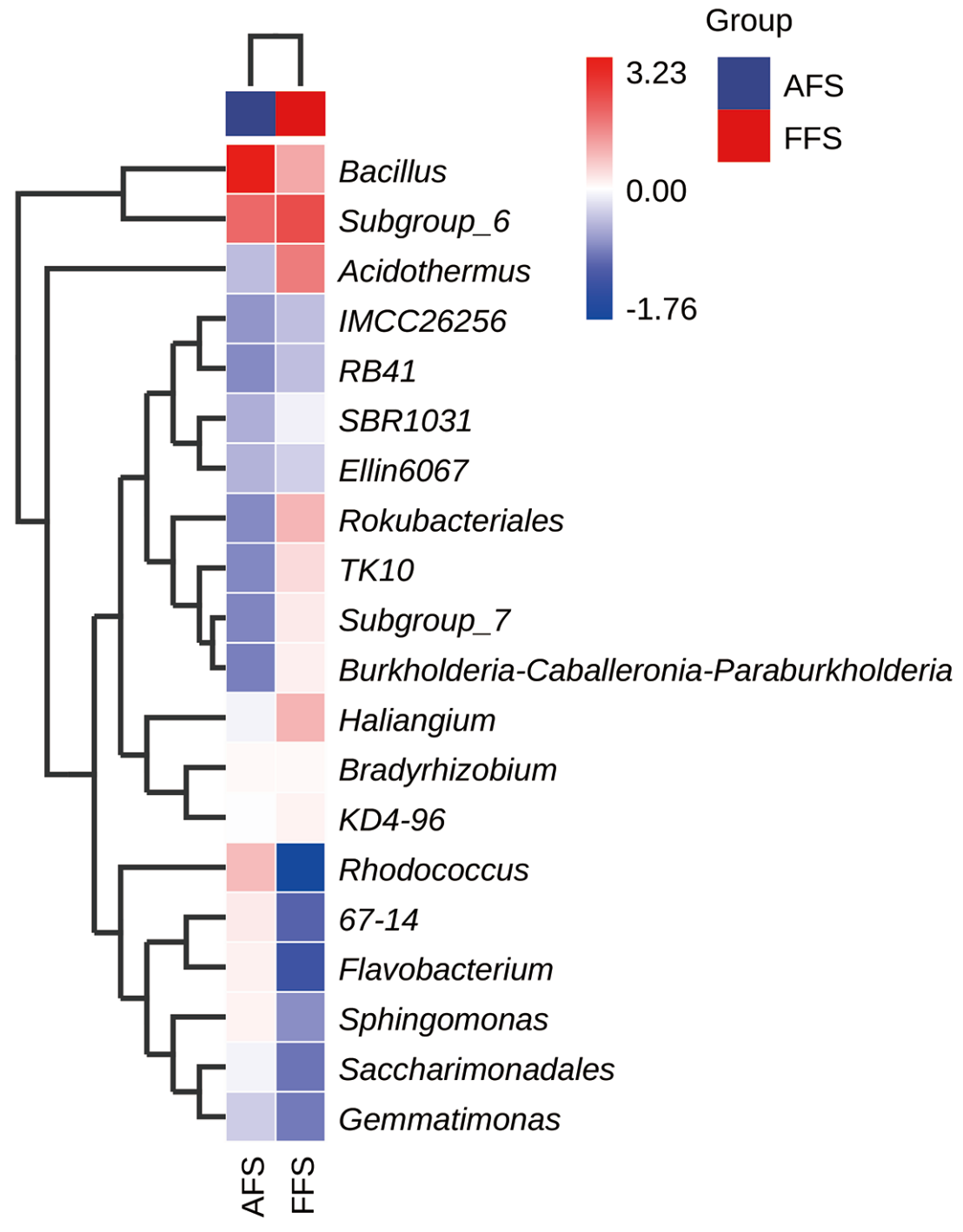




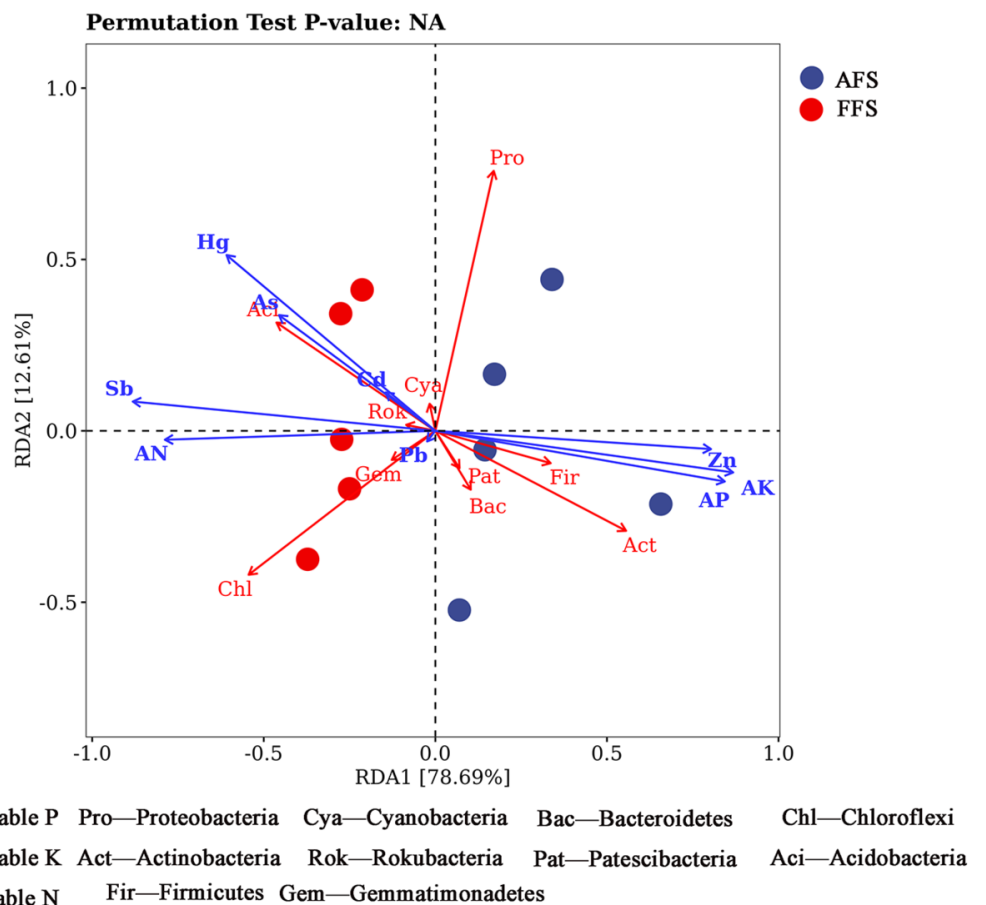

